ISSN 0001-6002/2003/45/2/80-83

Acta Médica Costarricense, $₫ 2003$

Colegio de Médicos y Cirujanos

\title{
Síndrome de Rapunzel. Reporte de un caso.
}

\author{
María Aguilar-Arauz ${ }^{1}$, Fernando Rodríguez-Gutiérrez², Christian Hernández-Mena ${ }^{3}$.
}

Se reporta el caso de una niña de tres años con pérdida de peso, anemia ferropénica y síntomas digestivos de varios meses de evolución, quien presentó un episodio de dolor y distensión abdominal intenso, que requirió manejo quirúrgico. Se evidenció la presencia de un tricobezoar asociado con un síndrome de Rapunzel.

El síndrome de Rapunzel consiste en la formación de una cola de pelo extendida desde estómago hacia intestino, lo que produce un efecto de acordeón en las asas intestinales, que lleva a necrosis isquémica.

Los bezoares, son cuerpos extraños en el tracto gastrointestinal, que pueden ocurrir como resultado de la ingesta de objetos o comidas que no atraviesan el píloro, aumentan de tamaño al sumarse fibras y comida no absorbible.'El término se origina a partir de badzher del árabe, padzhar del persa y beluzaar del hebreo, que significan antídoto 2 , ya que en culturas antiguas las piedras o sustancias duras que se encontraban en los estómagos o intestinos de los animales se consideraban sagradas, y se decía tenían poderes curativos.

Los bezoares se han observado en mujeres con problemas de personalidad o en individuos portadores de desequilibrios psiquiátricos. Usualmente se desarrollan como complicaciones de cirugía gástrica que alteran la función pilórica, o en circunstancias en que disminuyan la motilidad gástrica o disminuyen la acidez del estómago ${ }^{3}$. Se dividen en varias categorías según el material constituyente: fitobezoares, tricobezoares, lactobezoares, bezoares de levaduras, bezoares como secuelas de cirugía gástrica, concreciones de materiales inorgánicos (asfalto, arena, goma), entre otros.

Descriptores: Bezoar, tricobezoar, Síndrome de Rapunzel, obstrucción intestinal, tricotilomania

Recibido: 18 de enero, 2003

Aceptado: 18 de marzo, 2003

Historia clínica: Femenina, de 3 años de edad, costarricense, vecina de Talamanca de Limón, quien estaba siendo tratada en consulta externa de medicina general en el EBAIS correspondiente, por un cuadro clínico de varios meses de evolución, caracterizado por: náuseas, vómitos, irritabilidad, astenia, dolor abdominal difuso, pérdida de peso y malestar general, sin episodios de diarrea, estreñimiento o sangrado digestivo. No se conoce historia de trastornos conductuales relacionados con pica o tricotilomanía; tampoco se reportaron evidencias de síndrome de niño agredido u otras alteraciones del comportamiento. Se observó retraso en los parámetros antropométricos, asociado con anemia microcítica hipocrómica severa, del orden de $6 \mathrm{mg} / \mathrm{dL}$ de hemoglobina. Se trató con antiparasitarios y dosis de hierro intramuscular, sin

Médico General EBAIS Paso Ancho 2., Área de Salud Paso Ancho San Sebastián.

2 Médico Asistente Especialista Servicio de Patología del Hospital Tony Facio, Caja Costarricense Seguro Social.

Médico General Clínica Carlos Durán.

Correspondencia: María Aguilar Arauz. Dirección: Jardines de Tibas, manzana L casa \# 7, Llorente, Tibas,San José.

E-mail: maria_aguilar_Arauz@yahoo.com obtenerse mejoría clínica. De manera súbita, la paciente presentó una exacerbación de su dolor abdominal, asociado con distensión, que la llevaron a consultar al servicio de urgencias del Hospital local, donde fue valorada por cirugía pediátrica. En el examen físico, como hallazgo importante, se destacó que la paciente estaba hidratada, taquipneica, consciente, con el abdomen distendido, con peristalsis aumentada, blando, depresible, doloroso intensamente a la palpación, sin signos de irritación peritoneal. Se le realizó hemograma en el que se encontró anemia grave (Hb: $6.9 \mathrm{mg} / \mathrm{dl}$, HTO: $26.6 \%$ ), con fórmula blanca dentro de límites normales. Se tomó radiografía simple de abdomen, sin obtenerse hallazgos concluyentes. En vista de la severidad y persistencia de los síntomas, se indicó un tránsito gastrointestinal, que mostró la cámara gástrica ubicada hacia arriba y al lado derecho, sin compresiones extrínsecas en incidencia lateral, con el resto de la anatomía dentro de límites normales; intraluminalmente, en el estómago había un defecto de llenado redondeado muy sugestivo de bezoar (Figura 1). El ultrasonido de abdomen, no evidenció ningún hallazgo en particular. Se decidió realizar laparotomía exploratoria, que permitió una gastrotomía con extracción de tricobezoar, quedando en el extremo distal hacia el píloro, un hilo grueso rodeado de material

80 AMC, Abril-Junio 2003, vol 45 (2) 


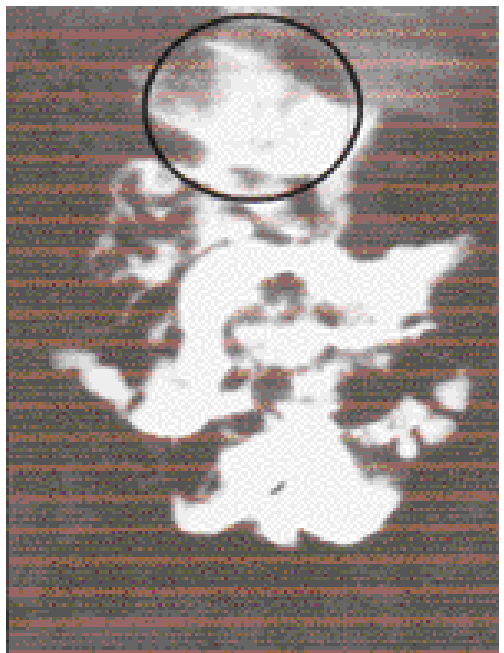

Figura 1: Radiografía de abdomen de pie en incidencia anteroposterior, parte del tránsito gastrointestinal, en la que se evidencia una imagen con defecto de llenado en el nivel de estómago.

vegetal que no se pudo extraer (Figura 2). En el nivel de yeyuno se encontraron 3 perforaciones rodeadas por el mismo hilo descrito anteriormente, por lo que se hizo necesaria la resección de $40 \mathrm{~cm}$ de ileon, que contenía otras tres perforaciones.

Durante el postoperatorio, la niña presentó varios picos febriles sin foco séptico al examen físico y sin alteración de laboratorios, los cuales resolvieron espontáneamente. Fue valorada por trabajo social, con el fin de iniciar estrategias de educación, seguimiento y control, para evitar tricofagia, así como los cuidados correspondientes sobre dieta, entre otras recomendaciones. En las visitas subsecuentes a consulta externa, la paciente no ha presentado a la fecha ninguna otra complicación. En el diagnóstico patológico e histológico se reportaron múltiples ulceraciones del borde antimesentérico, con, necrosis isquémica del intestino delgado en la pieza resecada (Figura 3).

\section{Discusión}

Los tricobezoares son concreciones gástricas compuestas del propio pelo del paciente, frecuentes en niños o mujeres jóvenes que se comen su propio cabello (tricofagia). Suelen formar un molde en el estómago. El cabello ingerido siempre se vuelve de color negro, independientemente de su color, debido a la desnaturalización de las proteínas por el ácido del jugo gástrico. Tricotilomanía es la tendencia compulsiva a arrancarse el pelo. Uno de cada 2000 niños sufre de tricotilomanía, muchos de tricofagia, pero pocos presentan tricobezoares $^{4,5,6}$. Esta conducta no es evidencia para pensar en tras-

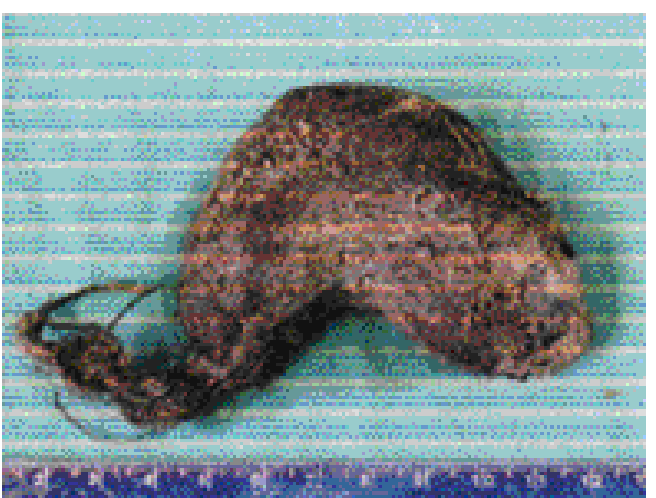

Figura 2:Tricobezoar extraído del estómago de la paciente. Se observa una cola de pelo que se desarrolla a partir del tricobezoar y se proyecta hacia intestino.

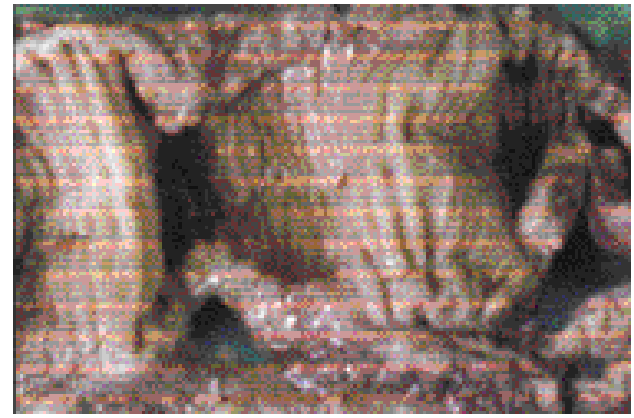

Figura 3: Pieza anatomopatológica que demuestra varias perforaciones en el nivel de íleon.Nótese la regularidad de dichas perforaciones en el nivel del borde antimesentérico, y el aspecto de acordeón que le ofrecen al intestino.

tornos mentales, más bien se asocia con trastornos psicológicos de poca importancia. Algunos autores refieren que si los pacientes menores se evalúan adecuadamente, todos presentan algún tipo de alteración psiquiátrica, que puede variar desde la ansiedad crónica hasta el retardo mental, mientras que otros hablan de que solo un $10 \%$ de los casos presenta una alteración psicológica ${ }^{8}$. La mayoría de las veces se observan en mujeres menores de 30 años (en el $85 \%$ de los casos) ${ }^{4,7}$. El pico de edad de comienzo de los síntomas es en el segundo decenio de la vida ${ }^{9}$. El diagnóstico es un reto, ya que el paciente niega u oculta la información sobre tricofagia $^{6}$, como se presenta en este caso, donde no había historia de tenencia de animales u otra fuente para el consumo de pelo. Aunque es infrecuente, la presencia de cabello en la materia fecal o en el vómito, permite hacer el diagnóstico ${ }^{4,11,12}$, pero lo anterior tampoco se documentó como hallazgo. El caso en cuestión escapa a lo descrito en la bibliografía consultada, puesto que la edad de la paciente no corresponde con el 
grupo etario más frecuente y no tiene historia de alteraciones conductuales o antecedentes de cirugías; se trata de un trastorno a muy temprana edad. Los tricobezoares usualmente se presentan con síntomas de obstrucción parcial de la salida del estómago o intestino. Se presenta saciedad precoz, hiporexia, náuseas y vómitos $(64 \%)$, hematemesis $(61 \%)$, pérdida de peso $(38 \%)$ y diarrea o constipación $(32 \%)^{4,6,9,13}$. Otros signos con los que se puede manifestar son: halitosis por putrefacción del material en el estómago ${ }^{7}$, masa móvil en el epigastrio (70\%), pérdida de pelo (alopecia en parches, o alopecia de cejas y pestañas), sangre oculta en heces y anemia ferropénica. La presencia de los síntomas depende de la elasticidad del estómago, el tamaño del bezoar y la aparición o no de complicaciones. La paciente presentó la mayoría de los síntomas y signos anotados, incluida la anemia ferropénica, con excepción de pérdida de pelo (no había alopecia en parches), diarrea o constipación y masa epigástrica. Lamerton ha descrito la palpación del bezoar por el tacto rectal en niños, además de palpar una masa firme en el cuadrante superior izquierdo ${ }^{6}$, empero no hay descripción de tacto rectal en el examen físico que se anotó.

Entre otros factores en la génesis de la formación de tricobezoares, están la longitud del cabello, las cantidades de pelo ingeridas, la disminución del peristaltismo, la alteración de la mucosa, la secreción ácida y el contenido de grasa en la dieta.

Las complicaciones de los bezoares, en este caso los tricobezoares, son mecánicas y traumáticas. Entre las primeras se encuentran la obstrucción y el íleo, y entre las traumáticas, la ulceración, hemorragia, perforación y peritonitis. La obstrucción intestinal baja se presenta cuando fragmentos del tricobezoar pasan el píloro y se detienen en algún segmento del intestino, entidad infrecuente que se conoce como Síndrome de Rapunzel. Su nombre evoca el cuento de los hermanos Grima, quines en 1812 escribieron la historia sobre Rapunzel, una joven que lanzaba su larga cabellera para que su enamorado príncipe subiera a la torre donde se encontraba presa.

Se pueden producir obstrucciones intestinales por la extensión de fibras de cabellos desde el bezoar gástrico a duodeno, o incluso hacia colon transverso. Desde el punto de vista fisiopatológico, se sugiere que el cabello es atrapado en los pliegues gástricos ${ }^{10}$ (en el $80 \%$ de los casos en la curvatura menor), y retenido por una insuficiente superficie de fricción, formándose una masa de pelo que actúa como ancla, y emite una proyección a través del píloro y hacia el intestino delgado ${ }^{4,5,7}$.

La diferencia de presiones entre el borde mesentérico del intestino, relativamente fijo, comparado con el del borde antimesentérico, móvil, sumado a la tensión por el peristaltismo, produce un efecto de acordeón que lleva a necrosis isquémica y perforación de la pared gástrica y del borde antimesentérico del intestino delgado. Según la nota operatoria, los hallazgos encontrados en la pieza resecada corresponden con lo antes descrito ${ }^{10}$ (Ilustraciones 2 y 3 ). Una radiografía abdomi- nal simple puede sugerir la presencia de un bezoar, sin embargo, la realizada a la paciente no suministró datos concluyentes al respecto.

El diagnóstico se realiza con el estudio radiológico donde se visualiza el bario rodeando una masa que generalmente flota en la suspensión, lo cual, además, lo diferencia de un tumor maligno6. También se visualiza cómo el bario queda atrapado en el intersticio del bezoar, dando una imagen de "superficie en panal de abejas". El diagnóstico sugestivo de bezoar se logró mediante el estudio baritado (Ilustración 1), con descripción similar a la mencionada. La ecografía permite diagnosticar bezoares cuando se observa con una banda ancha de ecos de gran amplitud con sombra acústica posterior en la luz gástrica ${ }^{6}$. Sin embargo, en el estudio ecográfico no se describieron datos similares. La especificidad del examen puede aumentarse pidiendo al paciente que ingiera líquido, observando que las burbujas se mueven en el interior de la masa; tal vez si se hubiera realizado esta maniobra, el ultrasonido hubiera sido de mayor utilidad.

La endoscopía digestiva es el método diagnóstico de elección ya que se ha demostrado que con la radiografía de las vías digestivas solo se diagnostica una cuarta parte de los casos identificados endoscópicamente ${ }^{9,11}$. A la vez sirve para la disgregación y extracción terapéuticas del material. Si la endoscopía no tiene éxito, puede ser necesaria la inter vención quirúrgica. En este caso se omitió la endoscopía por razones de equipo (no se contaba con gastroscopio pediátrico) y se procedió con la intervención quirúrgica. El objetivo del tratamiento es la remoción y prevención de la recurrencia.

La tricofagia exige un tratamiento psiquiátrico adecuado, de lo contrario no son infrecuentes las recidivas del tricobezoar (20\% de los casos) $)^{9,11,13}$. El tratamiento puede ser no quirúrgico, mediante el empleo de enzimas tales como las celulasas vía oral, que consiguen la disolución del bezoar; puede recurrirse al lavado agresivo a través de una sonda o bien a la fragmentación endoscópica combinada o no con inyección enzimática en la masa, esta última es tediosa porque se necesitan muchas sesiones. Cuando se encuentra asociado a una úlcera, no es necesario hacer tratamiento quirúrgico de esta, ya que al retirar el bezoar, mejora la lesión.

Los bezoares indisolubles deben ser removidos mecánicamente, ya sea por endoscopía o por cirugía. Pueden ser fragmentados con litotripsia extracorpórea con onda de choque, mas si el tamaño del bezoar es muy grande, se debe remover quirúrgicamente. El tratamiento quirúrgico presenta una baja mortalidad, que en la revisión de DeBakey fue del 5\% ${ }^{1}$. En casos de Síndrome de Rapunzel, se realiza una gastrotomía y si hay compromiso del intestino delgado, múltiples enterotomías ${ }^{4,5,6,7,9}$; si hay una obstrucción del intestino delgado secundaria a un bezoar, siempre se debe explorar el estómago para descartar un bezoar gástrico ${ }^{9}$.

\section{AMC, Abril-Junio 2003, vol 45 (2)}




\section{Abstract}

We report the case of a 3-year-old female who for several months had unespecific digestive symptoms, weigth loss and iron deficiency anaemia. She suffered an acute abdominal pain episode associated with tenderness that required an exploratory laparotomy. A thrichobezoar was found in the stomach which was associated with a Rapunzel Syndrome.

Rapunzel Syndrome consists in the formation of a hair- ball or trichobezoar that later develops a hairy tail that extends through the bowel. This produces an achordeon effect resulting in bowel necrosis.

\section{Referencias}

1. Debakey P, Ochscher A. Bezoar and concretions. Surgery 1938; 4:934963.

2. Singla S L, Rattan KN, Kaushik N, Pandit SK. Rapunzel Syndrome A case report. Am J Gastroenterol 1999; 94(7):1970-1971.

3. Phillips M R, Zalman Z,Drugas G. Case report and Literature Review. Mayo Clin Proc 1998; 73(7):653-656.

4. Delypere J P, Praet M, Verdonk G. An unusual case of Trichobezoar: The Rapunzel Syndrome. Am J Gastroenterol 1982; 77(7):467-470.

5. Pul N, Pul M. The Rapunzel Syndrome ( trichobezoar) Causing gastric perforations in a Child:a case report. Eur J Pediatr 1996; 155(1):18-19.

6. Pozo JC, Gómez TA, Rincón N, Berrios C. Tricobezoar: Diagnóstico inusual: Reporte de 3 casos. GEN 1995; 49(2):157-160.

7. Goldstein S, Lewis J, Rothstein R. Intestinal Obstruction Due to Bezoars. Am J Gastroenterol 1984; 79(4):313-318.

8. Rao P L, Mitra S K, Pathak I C. Trichobezoar in Children. Int Surg 1981; 66(1):63-65.

9. Andrus C, Ponsky J. Bezoars: Clasification, Pathophysiology and and tratement. Am J Gastroenterol 1988; 83(5): 467-468.

10. Lee J. Bezoares y cuerpos extraños en el estóma go. Clin Endosc North Am 1996; 3: 573-577.

11. Robles R, Parrilla P, Escamilla C. Gastrointestinal Bezoars. Br J Surg 1994; 81:1000-1001.

12. Seker B, Diler O N, Karaaybaz M. Trichobezoars as a Cause of Gastrointestinal Obstructions: The Rapunzel Syndrome. Acta Gastroenterol Bel 1996; 59(2):166-167.

13. Rider J, Forestl F, Griido J. Treatment and Prevention. Am J Gastroenterol 1984; 79(5):357. 